\title{
Impact of Awareness on the Spread of Dengue Infection in Human Population
}

\author{
Sunita Gakkhar ${ }^{1}$, Nareshkumar C. Chavda ${ }^{2}$ \\ Department of Mathematics, Indian Institute of Technology, Roorkee, India \\ Email: sungkfma@gmail.com, anchavda@yahoo.com \\ Received May 7, 2013; revised June 7, 2013; accepted June 14, 2013
}

Copyright (C) 2013 Sunita Gakkhar, Nareshkumar C. Chavda. This is an open access article distributed under the Creative Commons Attribution License, which permits unrestricted use, distribution, and reproduction in any medium, provided the original work is properly cited.

\begin{abstract}
In this paper, a model is proposed to study the impact of awareness on the dynamics of dengue. It is assumed that due to awareness of the disease some susceptible take necessary precautionary measures to protect themselves from mosquito bite. A threshold is obtained for the stability of the disease-free equilibrium state. The awareness is found to affect the threshold. For the sufficiently large awareness rate, the endemic state does not exist and disease-free state remains globally stable. It is concluded that the increase in the awareness rate decreases the densities of infectious populations of human as well as mosquitoes.
\end{abstract}

Keywords: Awareness; Basic Reproduction Number; Stability

\section{Introduction}

Dengue is viral infection transmitted by the bite of an infected Aedes mosquito causing severe flu-like symptoms which may lead to potentially lethal complications. The disease affects infants, children and adults and could be fatal. The symptoms of dengue fever vary according to the age of the patient. Once infected, the human becomes the main carrier and multiplier of the virus, serving as a source of the virus for uninfected mosquitoes. The virus circulates in the blood of an infected person for 2 - 7 days, at approximately the same time that the person develops a fever. Patients who are already infected with the dengue virus can transmit the infection via Aedes mosquitoes after the first symptoms appear (during 4 - 5 days; maximum 12). Symptoms usually last for 2 - 7 days, after an incubation period of 4 - 10 days after the bite from an infected mosquito [1,2]. The only method of controlling or preventing dengue virus transmission is to effectively control the mosquitoes and their bites.

There are four distinct serotypes of the dengue virus (DEN 1, DEN 2, DEN 3 and DEN 4). In humans recovery from infection by one dengue virus provides lifelong immunity against that particular virus serotype. However, this immunity confers only partial protection against sub-

\footnotetext{
${ }^{*}$ The first author (Sunita Gakkhar) acknowledges the finacial support provided by DST India for the reserach work Grant No. DST 612 MTD.
}

sequent infection by the other three serotypes of the virus. Once infected, a mosquito remains infected for life, transmitting the virus to susceptible individuals during probing and feeding.

Different mathematical models have been proposed to study the spread of dengue. Host vector type models are generally considered. The host (human) population consists of susceptible, exposed, infectious and removed compartments. For the vector (mosquito), only susceptible and infective compartments are considered. Feng and Vealsco proposed and model to study the population dynamics of vector transmitted disease with two pathogen strains [3]. A model for the transmission of dengue fever in a constant human population and variable vector population is discussed by Estava and Vargas [4]. The same authors in [5] proposed another model considering vertical and mechanical transmission in the vector population, to study the effects on the dynamics of the disease. Some more mathematical models have been developed in the literature to gain insights into the transmission dynamics of dengue in a community (see, for instance, [6-11]).

The spread of the disease in the population make the people to change their behavior so as to protect themselves from the disease. Such change of attitude and behavior can be considered as awareness towards the disease. When the dengue infected person gets recovered from the disease, he takes all the necessary precautions 
not to experience its bitterness again. For this, he takes precautionary measures such as use of mosquito nets, repellents etc., eliminating the possibility of mosquito bite and hence dengue infection. To incorporate this idea, a separate class of aware individual is taken into consideration. The infective will become aware after recovery. Also, transitions from susceptible to aware class directly are possible. This is due to the fact that infection of one member makes the whole family and friends aware to take necessary steps to be protected against the disease. The preventive measures due to awareness may bring vital changes in the dimension of the epidemic. Keeping these in view, a dynamic model incorporating the awareness is proposed for the dengue infection.

\section{Mathematical Model}

The most important and responsible factor for the spread of the dengue fever (DF) in the human population is the mosquito population. Therefore to study and predict the control of the DF it is necessary to consider the dimension of the mosquito population along with the human population. The total human population of interest is divided into three mutually disjoint classes. The members of susceptible class $\left(C_{1}\right)$ are at the risk of contracting DF. Each member of aware class $\left(C_{2}\right)$ is very much cautious to protect themselves from the mosquito bites and hence from dengue also. The members of dengue infected class $\left(C_{3}\right)$ are getting infection due to infected mosquito bite. Let $S(t), S_{h}(t)$ and $I(t)$ be the number of individuals in these three compartments respectively such that the total human population at time $t$ is $N(t)=S(t)+S_{h}(t)+I(t)$.

Let $M(t)$ be the total population of mosquitoes which is divided into susceptible $S_{1}(t)$ and infected population $I_{1}(t)$ at time $t$ such that $M(t)=S_{1}(t)+I_{1}(t)$. Further, for constructing the model the following assumptions are considered:

Human susceptible become dengue infected following bite of the dengue infected mosquito. The infected individual may have any of the four serotypes of dengue virus. Some susceptible are becoming aware and are taking sufficient protection to avoid mosquito bites. After recovery, the lifelong immunity is achieved to the particular serotype. It is assumed that after recovery the infected person becomes aware as he or she understands the risk factors for the disease. Further, due to awareness all protections will be used by the recovered individual and he or she will not be then infected by any other serotype of dengue virus. The decay in the life expectancy of human population due to dengue virus is ignored.

Let $\omega$ and $\omega_{1}$ be the constant recruitment rates of two populations while $D$ and $D_{1}$ be the natural death rates in host (human) and vector (mosquito) populations respectively. Let $\alpha_{1}$ be the dengue infection transmission coefficient from mosquito to human. The awareness is spread in the susceptible population at the rate $m_{1}$. This incorporates the direct transfer from susceptible to aware class. Infected human are treated and recovered at the rate $\gamma$.

The susceptible mosquitoes are infected with the virus while probing or feeding the dengue infected individual and the transmission coefficient is $\alpha_{2}$. The infected mosquito transmits the virus to the susceptible person on biting. No vertical transmission of virus is considered for the mosquito population. No recovery is assumed in the vector population. Accordingly, the SI model is considered for the mosquito population. These assumptions lead to the following model:

$$
\begin{aligned}
& \frac{\mathrm{d} S}{\mathrm{~d} T}=\omega-\alpha_{1} S I_{1}-m_{1} S-D S \\
& \frac{\mathrm{d} S_{h}}{\mathrm{~d} T}=m_{1} S+\gamma I-D S_{h} ; \\
& \frac{\mathrm{d} I}{\mathrm{~d} T}=\alpha_{1} S I_{1}-(\gamma+D) I \\
& \frac{\mathrm{d} S_{1}}{\mathrm{~d} T}=\omega_{1}-\alpha_{2} S_{1} I-D_{1} S \\
& \frac{\mathrm{d} I_{1}}{\mathrm{~d} T}=\alpha_{2} S_{1} I-D_{1} I_{1}
\end{aligned}
$$

All parameters are assumed to be nonnegative. The following nonnegative initial conditions are associated with the system (5.1):

$$
S(0)=S_{0}, S_{h}(0)=S_{h 0}, I(0)=I_{0}, S_{1}(0)=S_{10}, I_{1}(0)=I_{10}
$$

It is easy to establish that the solution of the IVP (5.1)(5.2) is positive. Further, the set

$$
\Omega=\left\{\left(S, S_{h}, I, S_{1}, I_{1}\right): S+S_{h}+I \leq \frac{\omega}{D}, S_{1}+I_{1} \leq \frac{\omega_{1}}{D_{1}}\right\}
$$

is found to be positive invariant.

Further, the dynamical system (5.1) can also be written as

$$
\frac{\mathrm{d} N}{\mathrm{~d} T}=\omega-D N, \frac{\mathrm{d} M}{\mathrm{~d} T}=\omega_{1}-D_{1} M
$$

It may be observed that $S_{h}$ does not appear explicitly in the system (5.1) except the second equation. Also, the limiting mosquito population is obtained as $S_{1}=\omega_{1} / D_{1}-I_{1}$. Accordingly, it is sufficient to consider only the first, third and the fifth equations to determine the dynamics of the system (5.1). Hence, the three-dimensional nonlinear system (5.4), given as follows, will be analyzed in the rest of this paper: 


$$
\begin{aligned}
& \frac{\mathrm{d} S}{\mathrm{~d} T}=\omega-\alpha_{1} S I_{1}-m_{1} S-D S \\
& \frac{\mathrm{d} I}{\mathrm{~d} T}=\alpha_{1} S I_{1}-(\gamma+D) I \\
& \frac{\mathrm{d} I_{1}}{\mathrm{~d} T}=\alpha_{2}\left(\frac{\omega_{1}}{D_{1}}-I_{1}\right) I-D_{1} I_{1}
\end{aligned}
$$

This system is positively invariant in the region

$$
\Omega_{1}=\left\{\left(S, I, I_{1}\right): 0 \leq S+S_{h}+I \leq \frac{\omega}{D}, 0 \leq I_{1} \leq \frac{\omega_{1}}{D_{1}}\right\}
$$

\section{Equilibrium Points and Basic Reproduction Number}

The non-linear dynamical system (5.4) always admits the disease-free equilibrium point $E_{0}\left(S^{*}, 0,0\right)$, where $S^{*}=\omega /\left(D+m_{1}\right)$. The basic reproduction number is obtained as the dominant eigenvalue of the next generation matrix [12]. It is important to distinguish new infections from all other class transitions in the population. Considering $X=\left(I, I_{1}, S\right)^{\mathrm{T}}$ where $I$ and $I_{1}$ are two infected classes, the system (5.4) can be written as

$$
\dot{X}=f(X) \Leftrightarrow \dot{X}=\mathcal{F}(X)-\mathcal{V}(X)
$$

The vector $\mathcal{F}$ is the rate of appearance of new infection in each class and $\mathcal{V}$ is the difference of transfers out of compartment and into the compartments:

$$
\mathcal{F}=\left[\begin{array}{c}
\alpha_{1} S I_{1} \\
\alpha_{2}\left(\frac{\omega_{1}}{D_{1}}-I_{1}\right) \\
0
\end{array}\right] ; \mathcal{V}=\left[\begin{array}{c}
(\gamma+D) I \\
D_{1} I_{1} \\
\alpha_{1} S I_{1}+m_{1} S+D S-\omega
\end{array}\right]
$$

The corresponding Jacobian matrices at disease freestate $E_{0}$ are computed as

$$
\begin{aligned}
\frac{\partial \mathcal{F}}{\partial X} & =\left[\begin{array}{cc}
F_{2 \times 2} & 0 \\
0 & 0
\end{array}\right] ; \frac{\partial \mathcal{V}}{\partial X}=\left[\begin{array}{cc}
V_{2 \times 2} & J_{1} \\
J_{2} & J_{3}
\end{array}\right] ; \\
F_{2 \times 2} & =\left[\begin{array}{cc}
0 & \frac{\alpha_{1} \omega}{\left(D+m_{1}\right)} \\
\frac{\alpha_{2} \omega_{1}}{D_{1}} & 0
\end{array}\right] ; V_{2 \times 2}=\left[\begin{array}{cc}
\gamma+D & 0 \\
0 & D_{1}
\end{array}\right]
\end{aligned}
$$

The basic reproduction number $R_{0}$ is obtained as the spectral radius of the next generation matrix $F V^{-1}$ and is computed as

$$
R_{0}^{2}=\frac{\alpha_{1} \alpha_{2} \omega \omega_{1}}{D_{1}^{2}(D+\gamma)\left(D+m_{1}\right)}
$$

The endemic equilibrium $E_{1}\left(\bar{S}, \bar{I}, \bar{I}_{1}\right)$ is obtained by solving the system:

$$
\begin{aligned}
& \omega-m_{1} S-\alpha_{1} S I_{1}-D S=0 ; \alpha_{1} S I_{1}-(\gamma+D) I=0 ; \\
& \alpha_{2}\left(\omega_{1} / D_{1}-I_{1}\right) I-D_{1} I_{1}=0
\end{aligned}
$$

This gives

$$
\begin{aligned}
E_{1}\left(\bar{S}, \bar{I}, \bar{I}_{1}\right) & \left(\frac{D_{1}\left(\alpha_{2} \bar{I}+D_{1}\right)(D+\gamma)}{\alpha_{1} \alpha_{2} \omega_{1}}, \frac{\alpha_{2} \omega_{1} \bar{I}}{D_{1}\left(\alpha_{2} \bar{I}+D_{1}\right)},\right. \\
& \left.\frac{\alpha_{1} \alpha_{2} \omega \omega_{1}-D_{1}^{2}(D+\gamma)\left(D+m_{1}\right)}{\alpha_{2}(D+\gamma)\left\{\alpha_{1} \omega_{1}+D_{1}\left(D+m_{1}\right)\right\}}\right)
\end{aligned}
$$

Since $\bar{I}>0$, the unique endemic equilibrium point exists when

$$
R_{0}>1
$$

Further, the equilibrium level of dengue infected human population $\bar{I}$ decreases if $m_{1}$ increases. Hence, the awareness reduces the severity of the epidemic.

\section{Stability Analysis}

The local stability of different equilibrium points of (5.4) can be discussed on the basis of stability matrix at $E\left(s, i, i_{1}\right)$. It is computed as

$$
J(E)=\left[\begin{array}{ccc}
-\left(\alpha_{1} i_{1}+D+m_{1}\right) & 0 & -\alpha_{1} s \\
\alpha_{1} i_{1} & -(D+\gamma) & \alpha_{1} s \\
0 & \alpha_{2}\left(\frac{\omega_{1}}{D_{1}}-i_{1}\right) & -\left(\alpha_{2} i+D_{1}\right)
\end{array}\right]
$$

The stability results are stated below in the form of theorems.

Theorem 4.1. The disease-free state $E_{0}$ is locally asymptotically stable when

$$
R_{0}<1
$$

Proof: The characteristic equation of stability matrix at $E_{0}\left(S^{*}, 0,0\right)$ is obtained as

$$
\begin{gathered}
\left(\lambda+D+m_{1}\right)\left(\lambda^{2}+a_{1} \lambda+a_{2}\right)=0 ; a_{1}=D+D_{1}+\gamma>0 ; \\
a_{2}=\left(D_{1}^{2}(D+\gamma)\left(D+m_{1}\right)-\alpha_{1} \alpha_{2} \omega \omega_{1}\right) /\left(D_{1}\left(D+m_{1}\right)\right)>0
\end{gathered}
$$

Therefore, the characteristic equation has roots with negative real parts for $R_{0}<1$.

Hence, the result is proved.

Remark 1. Clearly, when $R_{0}<1$ there will be no disease and when $R_{0}>1$ disease will persist in the population. Note that the awareness $m_{1}$ decreases the basic reproduction number $R_{0}$, thereby increasing the stability of $E_{0}\left(S^{*}, 0,0\right)$. It can also be observed that $R_{0}$ decreases as the natural death rates $D$ and $D_{1}$ increase.

Remark 2. It may be noted from (5.7) and (5.8) that the 
local stability of $E_{0}$ ensures the non-existence of endemic equilibrium point $E_{1}$. This suggests that the disease-free state $E_{0}$ may be globally stable when $R_{0}<1$. This is proved by using the geometric approach [13-15] for global stability in the next theorem.

Theorem 4.2. The locally stable disease-free equilibrium, $E_{0}\left(S^{*}, 0,0\right)$ is globally asymptotically stable.

Proof: The Jacobean matrix and second additive compound matrix of the system (5.4) around the equilibrium point $E_{0}\left(S^{*}, 0,0\right)$ are obtained as

$$
\begin{aligned}
& J=\left[\begin{array}{ccc}
-\left(D+m_{1}\right) & 0 & -\frac{\alpha_{1} \omega}{D+m_{1}} \\
0 & -(D+\gamma) & \frac{\alpha_{1} \omega}{D+m_{1}} \\
0 & \frac{\alpha_{2} \omega_{1}}{D_{1}} & -D_{1}
\end{array}\right], \\
& J^{(2)}=\left[\begin{array}{ccc}
-\left(2 D+\gamma+m_{1}\right) & \frac{\alpha_{1} \omega}{D+m_{1}} & \frac{\alpha_{1} \omega}{D+m_{1}} \\
\frac{\alpha_{2} \omega_{1}}{D_{1}} & -\left(D+D_{1}+m_{1}\right) & 0 \\
0 & 0
\end{array}\right]
\end{aligned}
$$

Define a function $P\left(S, I, I_{1}\right)=\operatorname{diag}\{S, S, S\}, P \in C^{1}$ and let $f$ be a vector field of (5.4), it may be noted that

$$
P_{f} P^{-1}=\operatorname{diag}\left\{\frac{\dot{S}}{S}, \frac{\dot{S}}{S}, \frac{\dot{S}}{S}\right\} \text { and } P J^{(2)} P^{-1}=J^{(2)}
$$

Taking $B=P_{f} P^{-1}+P J^{(2)} P^{-1}$, where the block matrix $B$ is given as

$$
\begin{gathered}
B=\left[\begin{array}{ll}
B_{11} & B_{12} \\
B_{21} & B_{22}
\end{array}\right], \text { with } \\
B_{11}=-\left(2 D+\gamma+m_{1}\right)+\frac{\dot{S}}{S}, \quad B_{12}=\left[\begin{array}{ll}
\frac{\alpha_{1} \omega}{D+m_{1}} & \frac{\alpha_{1} \omega}{D+m_{1}}
\end{array}\right], \\
B_{21}=\left[\begin{array}{c}
\frac{\alpha_{2} \omega_{1}}{D_{1}} \\
0
\end{array}\right], B_{22}=\left[\begin{array}{cc}
A-m_{1} & 0 \\
0 & A-\gamma
\end{array}\right], A=-\left(D+D_{1}\right)+\frac{\dot{S}}{S}
\end{gathered}
$$

Let $\mu$ denote the Lozinski measure, then it is computed as:

$$
\begin{aligned}
& \mu(B) \leq \max \left\{g_{1}, g_{2}\right\} \\
& =\max \left\{\mu\left(B_{11}\right)+\left|B_{12}\right|, \mu\left(B_{22}\right)+\left|B_{21}\right|\right\} \\
& g_{1}=\frac{\dot{S}}{S}-\left(2 D+\gamma+m_{1}\right)+\frac{\alpha_{1} \omega}{D+m_{1}}, \\
& g_{2}=\frac{\dot{S}}{S}-\left(D+D_{1}+\gamma\right)+\frac{\alpha_{2} \omega_{1}}{D_{1}}
\end{aligned}
$$

Now, when $R_{0}<1$, there exists a number $w>0$ such that $\mu(B) \leq \dot{S} / S-w$ provided

$$
\begin{aligned}
& \left(1+\frac{D+m_{1}}{D+\gamma}\right)>\frac{\alpha_{1} \omega}{\left(D+m_{1}\right)(D+\gamma)}, \\
& \left(1+\frac{D_{1}+\gamma}{D_{1}}\right)>\frac{\alpha_{2} \omega_{1}}{D_{1}^{2}}
\end{aligned}
$$

Hence, $\frac{1}{t} \int_{0}^{t} \mu(B) \mathrm{d} s \leq \frac{1}{t} \frac{\log (S)}{S_{0}}-w$.

Combining the two inequalities in (5.9) gives the condition

$$
\left(1+\frac{D+m_{1}}{D+\gamma}\right)\left(1+\frac{D_{1}+\gamma}{D_{1}}\right)>\frac{\alpha_{1} \omega \alpha_{2} \omega_{1}}{D_{1}^{2}\left(D+m_{1}\right)(D+\gamma)}
$$

Observe that left side of inequality is always greater than unity while right side is less than unity. Therefore the condition is always true. Hence, the result is proved.

Theorem 4.3. The endemic state $E_{1}$ if exists, is locally asymptotically stable.

Proof: When $R_{0}>1$, the characteristic equation about $E_{1}$ is obtained as

$$
\begin{aligned}
& \left(\lambda^{3}+a_{0} \lambda^{2}+a_{1} \lambda+a_{2}\right)=0, \\
& a_{0}=2 D+D_{1}+\alpha_{2} I+\gamma+m_{1}+\frac{\alpha_{1} \alpha_{2} \omega \omega_{1}}{D_{1}\left(D_{1}+\alpha_{2} I\right)}>0 ; \\
& a_{2}=\left(\alpha_{2} I(D+\gamma)\left(\alpha_{1} \omega_{1}+D_{1}\left(D+m_{1}\right)\right)\right) / D_{1}>0, \\
& a_{1}=D^{2}+\left(D_{1}+\gamma\right) m_{1}+\alpha_{2} I\left(\gamma+m_{1}\right) \\
& +D\left(D_{1}+2 \alpha_{2} I+\gamma+m\right)+\frac{\alpha_{1} \alpha_{2} I\left(D+D_{1}+\alpha_{2} I+\gamma\right)}{D_{1}\left(D_{1}+\alpha_{2} I\right)}>0
\end{aligned}
$$

Since $a_{1} a_{2}>a_{3}$, the characteristic equation has roots with negative real parts. Hence, the result is proved.

Theorem 4.4. The endemic state $E_{1}$, if exists, is globally asymptotically stable if

$$
\begin{aligned}
& \alpha_{2} \omega_{1}\left(\alpha_{1} \alpha_{2} \omega_{1} I+D_{1}\left(\alpha_{2} I+D_{1}\right)\left(D_{1}+D+m_{1}\right)\right) \\
& >D_{1}^{2}\left(\alpha_{2} I+D_{1}\right)^{2} \eta, \\
& \left(D_{1}+D+\alpha_{2} I\right)+\min \left\{m_{1}, \gamma\right\}>\alpha_{2} \omega_{1} /\left(\alpha_{2} I+D_{1}\right)
\end{aligned}
$$

Proof: Denote $\eta=D+\gamma$, the Jacobian matrix and second additive compound matrix of the system (5.4) around $E_{1}\left(\bar{S}, \bar{I}, \bar{I}_{1}\right)$ are

$$
J=\left[\begin{array}{ccc}
-\left(\sigma+D+m_{1}\right) & 0 & -\frac{D_{1} \rho_{1} \eta}{\alpha_{2} \omega_{1}} \\
\sigma & -\eta & \frac{D_{1} \rho_{1} \eta}{\alpha_{2} \omega_{1}} \\
0 & \alpha_{2}\left(\frac{\omega_{1}}{D_{1}}-\frac{\alpha_{2} \omega I}{D_{1} \rho_{1}}\right) & -\rho_{1}
\end{array}\right]
$$




$$
\begin{gathered}
J^{(2)} \\
=\left[\begin{array}{ccc}
-\left(\sigma+D+\eta+m_{1}\right) & \rho D_{1} \eta & \rho D_{1} \eta \\
1 / \rho & -\left(\eta+m_{1}+\rho_{1}+\sigma\right) & 0 \\
0 & -\left(\rho_{1}+\eta\right) & -\left(\eta+\rho_{1}\right)
\end{array}\right], \\
\quad \sigma=\frac{\alpha_{1} \alpha_{2} \omega_{1} I}{D_{1}\left(\alpha_{2} I+D_{1}\right)}, \rho=\frac{\rho_{1}}{\alpha_{2} \omega_{1}}, \rho_{1}=\alpha_{2} I+D_{1}
\end{gathered}
$$

Using the same approach as in Theorem (4.2), the matrix $B$ is computed as

$$
\begin{gathered}
B_{11}=-\left(\frac{\alpha_{1} \alpha_{2} \omega_{1} I}{D_{1}\left(\alpha_{2} I+D_{1}\right)}+D+\eta+m_{1}\right)+\frac{\dot{S}}{S}, \\
B_{12}=\left[\frac{D_{1}\left(\alpha_{2} I+D_{1}\right) \eta}{\alpha_{2} \omega_{1}} \frac{D_{1}\left(\alpha_{2} I+D_{1}\right) \eta}{\alpha_{2} \omega_{1}}\right] \\
B_{21}=\left[\begin{array}{c}
\left.\frac{\alpha_{2} \omega_{1}}{\left(\alpha_{2} I+D_{1}\right)}\right], \\
0
\end{array}\right] \\
B_{22}=\left[\begin{array}{cc}
-\left(m_{1}+\alpha_{2} I+\frac{\alpha_{1} \alpha_{2} \omega_{1} I}{D_{1}\left(\alpha_{2} I+D_{1}\right)}\right)+A \\
+\frac{\alpha_{1} \alpha_{2} \omega_{1} I}{D_{1}\left(\alpha_{2} I+D_{1}\right)} & -\left(\gamma+\alpha_{2} I\right)+A
\end{array}\right]
\end{gathered}
$$

The Lozinski measure $\mu(B)$ is computed as

$$
\begin{gathered}
\mu(B)=\max \left\{g_{1}, g_{2}\right\} \\
=\max \left\{\mu\left(B_{11}\right)+\left|B_{12}\right|, \mu\left(B_{22}\right)+\left|B_{21}\right|\right\} \\
g_{1}=\frac{\dot{S}}{S}-\left(\frac{\alpha_{1} \alpha_{2} \omega_{1} I}{D_{1}\left(\alpha_{2} I+D_{1}\right)}+D_{1}+D+m_{1}\right)+\frac{D_{1}\left(\alpha_{2} I+D_{1}\right) \eta}{\alpha_{2} \omega_{1}}, \\
g_{2}=\frac{\dot{S}}{S}-\left(D_{1}+D+\alpha_{2} I\right)+\frac{\alpha_{2} \omega_{1}}{\left(\alpha_{2} I+D_{1}\right)}+\max \left\{-m_{1},-\gamma\right\}
\end{gathered}
$$

If the conditions in (5.10) are satisfied then there exist a number $w>0$ such that

$$
\mu(B) \leq \frac{\dot{S}}{S}-w
$$

This establishes the result.

However, the endemic state may be globally stable even when these conditions are not satisfied.

\section{Numerical Simulations}

The following data set is chosen to confirm different analytical results:

$$
\begin{aligned}
& \alpha_{1}=0.00002, \alpha_{2}=0.000035, D=0.000045, \\
& D_{1}=0.05, \gamma=0.012, \omega=0.405, \omega_{1}=110
\end{aligned}
$$

For the above dataset with $m_{1}=0.002$ the basic reproduction number $R_{0}$ is computed as 0.506414 . Since $R_{0}$
$<1$, the disease free equilibrium point of the system (5.4) is computed as $(198,0,0)$. The solution of system (5.1) is numerically obtained for this data set and with different initial conditions. It was observed that the solution converges to the disease free state after removing the initial transients. The endemic state does not exist in this case. However, for $m_{1}=0.00025$ the basic reproduction number is computed as $R_{0}=3.5105$. In this case, the endemic state is computed as $(401,24,36)$. For these two values of awareness parameter $m_{1}$, the time series for susceptible, infected human and infected mosquitoes is obtained in Figures 1 and 2. These time series confirms the analytical results.

\section{Conclusion}

A five dimensional model is proposed and analyzed to get insights of dengue transmission in the human population. It is assumed that due to awareness of the disease some susceptible take necessary precautionary measures to protect themselves from mosquito bite and joins aware class. It is also assumed that all the recovered individuals are convinced that the dengue re-infection is very dangerous so they take sufficient care to protect themselves from the mosquito bites so they also join the aware class after recovery. Increase in the awareness decreases density of infected human as well as infected mosquitoes. Also, increase in the rate of awareness decreases basic

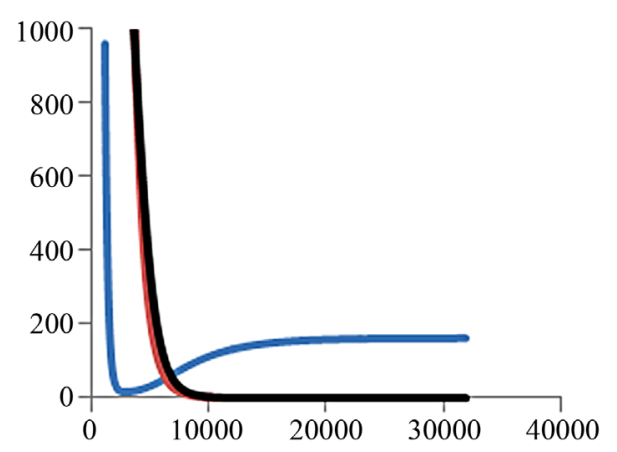

Figure 1. Time series for $m_{1}=0.002$. Blue: susceptible host, black: infective host, red: infective vector.

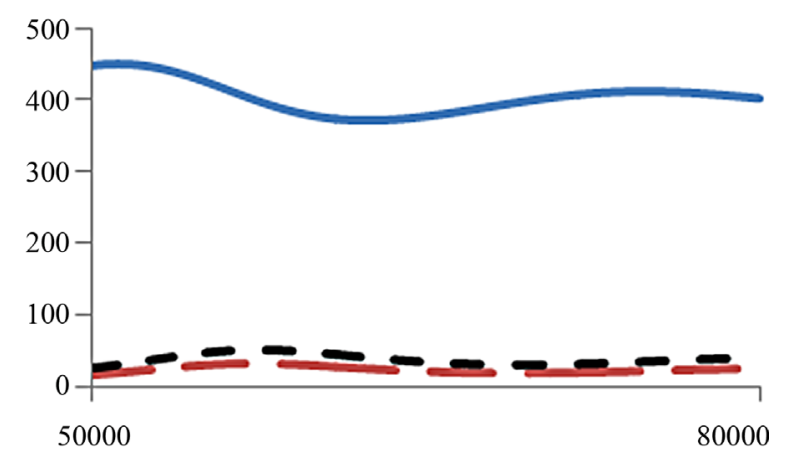

Figure 2. Time series for $m_{1}=\mathbf{0 . 0 0 0 2 5}$. Blue: susceptible host, black: infective host, red: infective vector. 
reproduction number. It also increases the stability of disease free state. Hence, it is concluded that sufficiently high rate of awareness plays a crucial role in reducing the menace of the disease.

\section{REFERENCES}

[1] "Dengue Guidelines of Diagnosis, Treatment, Prevention and Control," WHO Report, 2009.

[2] D. J. Gubler, "Dengue," In: T. P. Monath, Ed., The Arbovirus: Epidemiology and Ecology, Vol. 2, CRC, Boca Raton, 1986, p. 213.

[3] Z. Feng and V. Vealsco-Hernandez, "Competitive Exclusion in a Vector-Host Model for the Dengue Fever," Journal of Mathematical Biology, Vol. 35, No. 5, 1997, pp. 523-544. doi:10.1007/s002850050064

[4] L. Esteva and C. Vargas, "Analysis of a Dengue Disease Transmission Model," Mathematical Biosciences, Vol. 150, No. 2, 1998, pp. 131-151. doi:10.1016/S0025-5564(98)10003-2

[5] L. Esteva and C. Vargas, "Influence of Vertical and Mechanical Transmission on the Dynamics of Dengue Disease," Mathematical Biosciences, Vol. 167, No. 1, 2000, pp. 51-64. doi:10.1016/S0025-5564(00)00024-9

[6] F. A. B. Coutinho, M. N. Burattini, L. F. Lopez and E. Massad, "Threshold Conditions for a Nonautonomous Epidemic System Describing the Population Dynamics of Dengue," Bulletin of Mathematical Biology, Vol. 68, No. 8, 2006, pp. 2263-2282. doi:10.1007/s11538-006-9108-6

[7] M. Derouich and A. Boutayeb, "Dengue Fever: Mathematical Modelling and Computer Simulation," Applied Mathematics and Computation, Vol. 177, No. 2, 2006, pp. 528-544. doi:10.1016/j.amc.2005.11.031

[8] N. M. Ferguson, C. A. Donnelly and R. M. Anderson, "Transmission Dynamics and Epidemiology of Dengue:
Insights from Age-Stratified Sero-Prevalence Surveys," Philosophical Transactions of the Royal Society of London B, Vol. 354, No. 1384, 1999, pp. 757-768. doi:10.1098/rstb.1999.0428

[9] C. J. Struchiner, P. M. Luz, C. T. Codeco, F. C. Coelho and E. Massad, "Current Research Issues in MosquitoBorne Diseases Modelling," Contemporary Mathematics, Vol. 410, 2006, pp. 349-352. doi: $10.1090 /$ conm $/ 410 / 07736$

[10] J. J. Tewa, J. L. Dimi and S. Bowang, "Lyapunov Functions for a Dengue Disease Transmission Model," Chaos, Solitons and Fractals, Vol. 39, No. 2, 2009, pp. 936-941. doi:10.1016/j.chaos.2007.01.069

[11] H. M. Yang and C. P. Ferreira, "Assessing the Effects of Vector Control on Dengue Transmission," Applied Mathematics and Computation, Vol. 198, No. 1, 2008, pp. 401-413. doi:10.1016/j.amc.2007.08.046

[12] P. van den Driessche and J. Watmough, "Reproduction Numbers and Sub-Threshold Endemic Equilibria for Compartmental Models of Disease Transmission," Mathematical Biosciences, Vol. 180, No. 1-2, 2002, pp. 29-48. doi:10.1016/S0025-5564(02)00108-6

[13] M. Y. Li and J. S. Muldowney, "Global Stability for the SEIR Model in Epidemiology," Mathematical Biosciences, Vol. 125, No. 2, 1995, pp. 155-164. doi:10.1016/0025-5564(95)92756-5

[14] R. H. Martin Jr., "Logarithmic Norms and Projections Applied to Linear Differential Systems," Journal of Mathematical Analysis and Applications, Vol. 45, No. 2, 1974, pp. 432-454. doi:10.1016/0022-247X(74)90084-5

[15] B. Buonomo and D. Lacitignola, "On the Use of the Geometric Approach to Global Stability for Three Dimensional ODE Systems: A Bilinear Case," Journal of Mathematical Analysis and Applications, Vol. 348, No. 1, 2008, pp. 255-266. doi:10.1016/j.jmaa.2008.07.021 